La internacionalización en las relaciones interinstitucionales

Dominga Mazzeo, Judith Baudo

Revista ES (en y sobre Educación Superior)

Vol.1, N¹-2 / Fecha de publicación: 27/12/2021

e-ISSN: 2718-6539

https://revistas.unlp.edu.ar/ES/index

IIES - Facultad de Odontología

DOI: https://doi.org/10.24215/27186539e028

\title{
La internacionalización en las relaciones interinstitucionales
}

\author{
Internationalization in the interinstitutional activities
}

Internacionalização nas relações interinstitucionais

Odontóloga Dominga Mazzeo

Facultad de Odontología - UNLP

damazzeo@yahoo.com.ar

Judith Baudo

\section{Resumen}

Asistimos a un mundo donde las opciones para el desarrollo se plasman de manera conjunta y asociadas, y los países van dejando de lado las decisiones tomadas en soledad. 
La colaboración y la cooperación solidaria entre los seres humanos subyacen en cualquier emprendimiento, los que se producen cada vez más en el marco de un intercambio que enriquece a todas las partes involucradas. Las instituciones universitarias han tomado conciencia de esta realidad en la que el intercambio académico y estudiantil se encuentra en permanente crecimiento, y las vinculaciones institucionales -acuerdos y convenios- ya no son un "lustre", sino más bien un valor agregado a la calidad institucional.

En la actualidad la Internacionalización de la Educación Superior es una realidad; propiciarla, afianzarla, sostenerla y divulgar sus beneficios se ha convertido en una nueva función universitaria.

\section{Abstract}

We are witnessing a world where development options are reflected together and associated, and countries are setting aside decisions made in solitude.

Collaboration and solidarity cooperation among human beings underlie any entrepreneurship, which is increasingly produced within the framework of an exchange that enriches all parties involved. University institutions have become aware of this reality in which academic and student exchange is constantly growing, and institutional links - agreements and covenants - are no longer a "luster", but rather an added value to institutional quality.

Today, the Internationalization of Higher Education is a reality; promoting, entrenching, sustaining and disseminating its benefits has become a new university function.

\section{Resumo}


Assistimos a um mundo onde as opções de desenvolvimento se refletem de forma conjunta e em parceria, e os países deixam de lado as decisões tomadas sozinhos.

A colaboração e a cooperação solidária entre os seres humanos estão na base de qualquer empreendimento, que ocorre cada vez mais no quadro de um intercâmbio que enriquece todas as partes envolvidas. As instituições universitárias têm se dado conta dessa realidade em que o intercâmbio acadêmico e estudantil não para de crescer, e os vínculos institucionais convênios e convenções - não são mais uma "glosa", mas uma mais-valia de qualidade institucional.

Atualmente a Internacionalização do Ensino Superior é uma realidade; promovê-la, fortalecê-la, sustentá-la e divulgar seus benefícios tornou-se uma nova função da universidade.

\section{Palabras clave}

Internacionalización, Educación Superior, Relaciones Interinstitucionales.

\section{Keywords}

Internationalization, Higher Education, Relations Inter-Agency.

\section{Palavras chave}

Internacionalização, Ensino Superior, Relacionamentos Interinstitucional.

\section{Relaciones Institucionales}

La Educación Superior persigue entre sus objetivos la promoción, la difusión y la preservación de la cultura, la investigación científica y tecnológica y la 
creación artística. Cumple las funciones de formar profesionales: docentes e investigadores; difundir las ideas, las conquistas de la ciencia y las realizaciones artísticas por medio del ejercicio de la docencia; generar conocimiento por medio de la investigación; difundir los beneficios de sus actividades científicas y su acción cultural y social mediante la extensión universitaria. Asistimos a un mundo donde las opciones para el desarrollo se plasman de manera conjunta y asociadas, y los países van dejando de lado las decisiones tomadas en soledad. La colaboración y la cooperación solidaria entre los seres humanos subyacen en cualquier emprendimiento, los que se producen cada vez más en el marco de un intercambio que enriquece a todas las partes involucradas. Las instituciones universitarias han tomado conciencia de esta realidad en la que el intercambio académico y estudiantil se encuentra en permanente crecimiento, y las vinculaciones institucionales -acuerdos y convenios- ya no son un "lustre", sino más bien un valor agregado a la calidad institucional. En la actualidad la Internacionalización de la Educación es una realidad; propiciarla, afianzarla, sostenerla y divulgar sus beneficios se ha convertido en una nueva función universitaria.

La planificación, entonces, comienza a definirse "como un proceso de reflexiónacción de un grupo social que pretende desarrollar y alcanzar sus valores. Por medio: de la interpretación de los fenómenos del contexto donde se insertan. Y por medio de la investigación de posibles caminos para lograr su cumplimiento" (Forero, 1999: 10).

Interpretar el contexto pasa a significar, estudiar y reconocer las relaciones sociales, culturales, políticas, económicas, logísticas y ambientales, que inciden en los objetivos que se propone una comunidad, para poder diseñar las estrategias y la organización adecuada para alcanzar y sostener esos 
objetivos, privilegiando incluso la consolidación de las capacidades estratégicas de la organización social, sobre el concepto de plan estratégico.

Cuando hablamos de capacidades estratégicas nos estamos refiriendo al desarrollo de las competencias individuales y colectivas, a la flexibilidad de las estructuras, al cambio en la naturaleza de los procesos de acción y a la modificación de los comportamientos, en síntesis, a la cultura del cambio.

La Universidad Nacional de La Plata es una institución que se propone construir conocimientos en conjunto con otros, no solo transferirlos, es por eso que el objetivo general de esta Universidad en sus relaciones institucionales, es vincularla con otras Universidades, conformando e integrando redes de intercambio y construcción conjunta de carreras de grado y posgrado y de proyectos de investigación de interés para el desarrollo de nuestro país, de la región y el mundo. También es vincularla con los distintos estamentos del Estado, con las organizaciones de nuestra Sociedad Civil y con la micro, pequeña y mediana empresa, procurando en todos los casos la integración de la Universidad desde la región al mundo y su vinculación intensa y reconocida con los diversos campos institucionales. Se busca fortalecer los procesos de transferencia de conocimiento y producción de servicios universitarios útiles y necesarios para los diversos sectores de la Comunidad, la pequeña y mediana empresa y el Estado en la búsqueda de mejorar las políticas públicas y el desarrollo ambientalmente sustentable, fomentar la transferencia tecnológica y general, promover la innovación y el emprendedorismo en los estudiantes y graduados como tema de central importancia para el desarrollo de la región, difundir la ciencia en la comunidad y crear conciencia ambiental en general y sobre los procesos científico-tecnológicos. 
En esa línea, son objetivos específicos de las políticas de relaciones institucionales de la UNLP, incrementar la movilidad docente, científica y estudiantil en el sistema universitario, promoviendo la mejor formación de grado y posgrado y proyectos conjuntos académicos, de investigación y extensión. Además, la Universidad debe responder a las demandas institucionales crecientes que le hace la sociedad y sumar en diversidad, flexibilidad y articulación. Formar parte de la comunidad académica mundial, integrar redes universitarias formativas, científicas y solidarias que permita estar a la altura de este tiempo, y comprender los escenarios en los que toca evolucionar, movilizando estudiantes, profesores e investigadores en un intercambio productivo que sume siempre en la interacción; e integrar redes de instituciones no universitarias, comunitarias, profesionales especialmente de los graduados, siendo selectivos en la prioridad de la selección, conscientes que se pertenece y se construye un modelo basado en el esfuerzo de lograr la inclusión como prioridad. (Tauber F, 2018).

\section{Internacionalización}

La internacionalización de las Universidades no es un fenómeno nuevo, aunque en los últimos años se ha convertido en objeto de debates y manifiesta una nueva dinámica institucional. A lo largo de la historia se ha podido rastrear que en las Universidades antiguas los estudiantes hablaban el idioma latín y se movilizaban de un país a otro, e incluso la movilidad de profesores, estudiantes, saberes, disciplinas de la época, caracterizaron el surgimiento de las Universidades (Garcia Guadilla, 2005). Posteriormente, con la creación el Estado Moderno, la dinámica de las Universidades se modificó, disminuyó la 
dinámica territorial, y las nuevas Universidades adquirieron la finalidad de dar respuesta a problemas e intereses del contexto nacional, de esta forma se identifican distintos modelos de universidad, por ejemplo, el modelo Napoleónico (1909), el modelo de Humbolt (1919), el modelo Soviético (1918). Los estados europeos crean sus propias instituciones universitarias, y científicas, comenzando a publicar en sus propias lenguas y revistas especializadas (Ben David, 1977). En este momento histórico las dinámicas internacionales consistían en llevar la Universidad europea al resto del mundo (Garcia Guadilla, 2005). De esta forma, desde las primeras décadas del siglo XX "las grandes potencias habían desarrollado sus estrategias internacionales de acuerdo con las políticas de desarrollo colonial [con lo cual, los procesos de] transferencia de las prácticas científicas de la metrópolis a la periferia se hizo más intensa. Sus objetivos principales eran dos: la influencia cultural y la competencia con otras naciones" (Vessuri, 2007: 72). En la etapa posterior a la Segunda Guerra Mundial, el modelo de Universidad occidental se consolidó, y pasó a identificarse como el modelo institucional intelectual y profesional al que debían aspirar los países que querían modernizarse (Schwartzman, 2009). Muchas de las ex colonias que lograron independizarse luego de la segunda guerra mundial, mantuvieron la lengua colonial en sus Universidades, y el modelo hegemónico paso a ser el norteamericano, incentivado por Agencias Internacionales y fundaciones privadas que actuaban en los países en desarrollo comenzaron a competir por la atracción de estudiantes (Coleman \& Court, 1993; Gaillard and Gaillard 1999). Asimismo, las políticas de cooperación internacional en Ciencia y Tecnología, hasta la década de 1980, puntualizaron la "asistencia técnica" (Ragouet, Shinn y Waast, 1997; Rist, 2008), y surgieron instituciones dedicadas a formación e investigación en 
Universidades públicas con el objetivo de reducir las brechas de conocimiento y problemas de información, con diferentes resultados (Vessuri, 2013).

A partir de 1990 los sectores gubernamentales e instituciones de Educación Superior (ES) dieron inicio a programas de internacionalización, a través de la firma de convenios, la asistencia a congresos internacionales y la participación en alianzas y redes académicas (Didou, 2007). Este fenómeno ha sido analizado principalmente por autores con procedencia canadiense, europea y estadounidense, presentándose una escasez de trabajos de autores latinoamericanos, lo que trajo aparejado que, en la década de los años noventa, la internacionalización de las Universidades de la región Latinoamericana estuviera signada por la visión de la cooperación internacional promulgada por Estados Unidos o Canadá y por el proceso de Bolonia iniciado en el continente europeo. Dan cuenta de ello, Knight (1994) y De Wit (1995), quienes fueron los pioneros en definir la Internacionalización de la ES (IES) como "el proceso de integración de una dimensión internacional/intercultural en las funciones de la enseñanza, la investigación y el servicio de la institución" (Knight, 1994: 2). Esta definición es la más utilizada en los ámbitos académicos y gubernamentales ya que presenta a la internacionalización como un proceso intercultural, que no sólo se orienta a los Estados como tal sino también a diferentes grupos culturales de un país.

En la literatura latinoamericana se destacan diferentes factores contextuales que impulsan la internacionalización de la Universidad. En primer lugar, la demanda creciente por Educación Superior frente a las exigencias de la 'educación permanente' o 'educación de por vida', así como también ante el deterioro del valor de las credenciales educativas. En segundo lugar, la creciente importancia del conocimiento avanzado y la especialización en 
diferentes áreas del conocimiento, lo cual hace imposible que un país pueda, por sí mismo, producir, desarrollar y formar recursos humanos en todas las áreas disciplinarias. En tercer lugar, los procesos de integración de la Educación Superior y de homologación de titulaciones en diferentes partes del mundo (Brunner, 2005). Por ejemplo, en el contexto de globalización se hace referencia a la construcción de espacios regionales de Educación Superior, como son el proceso Bolonia (Hermo y Verger, 2012, García Barbero, 2010) o el Mercosur Educativo (Perrotta, 2011; Donini, 2010; Robledo y Caillón, 2009). Desde esta perspectiva Krotsch (1997: 21) entiende que "la regionalización constituye una forma de procesar la globalización en términos de cambios y transformación en los patrones normativos y de la vida social y económica de los países. Las Universidades ya no pueden optar por cooperar, crear interdependencias y configuraciones. Existen determinaciones estructurales que los obligan a ello".

De acuerdo a este enfoque, el fenómeno de la Internacionalización de la Educación Superior, y de la Universidad en particular, también está asociado al proceso de globalización económica al menos en dos sentidos.

1) La globalización implica la creciente internacionalización del sector de servicios y la libre distribución transnacional de personas, inversiones, ideas, valores y tecnologías, entre los que se halla la educación superior o educación trasnacional (Brunner, 1999 y Rama, 2003).

2) La globalización requiere la formación de cuadros profesionales capaces de actuar en la nueva realidad global y que respondan a las exigencias de educación permanente. En este sentido, la internacionalización de los universitarios permite el acceso a mayores y diferentes ámbitos de conocimiento y a la formación en una perspectiva global (Gacel-Ávila, 2000). 
En este marco, se considera que la internacionalización de las Universidades implica un nuevo estilo de gestión y un cambio en la cultura institucional. Es decir, se torna preciso repensar la misión de la Universidad, sus funciones y la relación entre sus componentes, abandonando la visión individual y adoptando una visión institucional de la dimensión internacional. Se hace necesaria la creación de políticas y estrategias institucionales específicas de internacionalización de la docencia, investigación y extensión, a partir del apoyo de las autoridades, de las estrechas relaciones entre los distintos actores de la comunidad universitaria y de un convencimiento general acerca de la importancia de la internacionalización de la institución (Gacel-Ávila, 2000). Desde una perspectiva' critica' se pueden identificar diferentes tipos de trabajos, que advierten sobre los efectos negativos que trae aparejada la Internacionalización de la Educación Superior.

1.- Por un lado, autores como Gascón y Cepeda (2004); Bernal (2007); y Yarzábal (2005) han identificado la internacionalización de la Educación Superior con la 'mercantilización de la educación', centrándose en la incorporación de la Educación Superior en el marco del Acuerdo General sobre Comercio de Servicios (GATS) de la Organización Mundial de Comercio (OMC) como un bien más a ser progresivamente liberalizado (Gascón y Cepeda, 2004; Bernal, s/f y Yarzábal, 2005). Para estos autores, dicha incorporación abriría las puertas a la conformación de una 'industria educativa a nivel mundial' de la mano de actores externos que en muchos casos tienen como único objetivo la ganancia, afectando negativamente a la calidad educativa, la pertinencia social de la educación y la democratización en el acceso. En este sentido, Yarzábal (2005) sostiene que, si bien la internacionalización de la Educación Superior es un fenómeno antiguo, existen distintas motivaciones de orden académico, 
político, económico, y socio-culturales que le otorgan sentido, ya que los distintos actores involucrados deciden el énfasis que adquiere cada una de las motivaciones que guían la internacionalización de la Educación Superior. El autor considera que el centro de gravedad de la internacionalización de la Educación Superior desde la Edad Media a la época contemporánea se ha desplazado desde motivaciones académicas, fundadas en el carácter internacional de las instituciones y la condición de bien públicos de los saberes transmitidos por ellas, hacia motivaciones económicas, que en el Hemisferio Occidental se propone impulsar sus modelos de desarrollo y competitividad (Yarzábal, 2005). Además, se denuncia al GATT como un marco regulatorio que promueven las corporaciones multilaterales y los gobiernos interesados en exportar servicios educativos (Altbach, 2004).

Por su parte, Carlos Tünnermann Bernheim (2008) dirige su crítica a la transnacionalización de la Universidad y hace referencia a que, si se plantea a la Educación Superior como un bien público global, deja de estar sujeta a las normas, regulaciones y jurisprudencia de los estados nacionales y pasa a estar regulada por la Organización Mundial del Comercio. Asimismo, diferencia la transnacionalización de la internacionalización: mientras que la transnacionalización se trata de facilitar la erradicación de filiales de universidades extranjeras en la Región, la internacionalización se basa en una cooperación solidaria y horizontal que respeta las identidades e idiosincrasia de los distintos países y, consecuentemente, se plantea como multicultural.

2.- Por otro lado, desde la perspectiva crítica se identifica la permanencia de un esquema convencional de distribución espacial de los flujos de estudiantes y de académicos en el extranjero desde el Sur hacia el Norte (Estados Unidos, España, Francia, Reino Unido y Alemania) identificando a la 
internacionalización de la Educación Superior, como una 'carnada' que utilizan los países del Norte para atraer a los mejores cerebros del Sur, con el fin de nutrir sus economías (Gascón Muro, Cepeda Dovala: 2009), y despojando a los países del Sur de uno de los bienes más preciados en el marco de las economías del conocimiento: los trabajadores del conocimiento (Gascón, 2008). Además del flujo de personas, Marginson; (2008) hace referencia a flujos de mensajes (y otro tipo de comunicaciones), flujos de información y conocimiento (incluyendo publicaciones y datos), flujos de capital financiero y recursos económicos, que se desplazan a través de relaciones diversas, verticales y horizontales, que pueden ser cooperativas o competitivas.

Distintos autores enfatizan en la necesidad de orientar la internacionalización hacia la integración de las Universidades Latinoamericanas desde una perspectiva solidaria y endógena (Krotsch; 1997; Didrikson, 2002; Rojas Mix, 2005; Naidof, 2005; Marcano, 2009; Siufi; 2009; Fernández Lamarra, 2009; Perrotta; 2012; Oregioni; 2014), a partir de resaltar la importancia de realizar actividades de cooperación horizontal entre instituciones y sectores, que se estructura en redes y en espacios comunitarios y trabaja en colaboración, sin perder su identidad institucional (Didrikson; 2008). Por ejemplo Krotsch (1997) y Didriksson (2002), focalizaron en el rol de los consorcios de Universidades para la integración universitaria. Borón (2008) menciona la importancia que han tenido las asociaciones de universidades en América Latina (destacando la Asociación de Universidades del Grupo Montevideo - AUGM), en señalar las catastróficas consecuencias del modelo neoliberal sobre las Universidades de la Región. En el mismo sentido, y en contraposición a los enfoques que sostienen que la integración en la década de los noventa fue exclusivamente comercial, Perrotta (2012) argumenta que la agenda educativa del Mercosur 
(una agenda no comercial) ha estado invisibilizada en el período 1992-2002, por no responder a los parámetros que exigía el pensamiento único ligado a una lógica mercantil en dicho momento histórico.

Además, se contempla la existencia de un denominador común entre las Universidades Latinoamericanas, que consiste en la necesidad de generar y transmitir conocimiento relevante a las necesidades sociales (Dagnino; 2007). Consecuentemente desde una perspectiva endógena se identifica a la Universidad como el ámbito apropiado para la gestión estratégica e integral (a partir de actividades de: docencia, investigación y extensión) de la internacionalización hacia Latinoamérica con el objetivo de enfrentar las connotaciones negativas del mundo globalizado, y generar conocimiento relevante. Es decir que en la Región existe resistencia a la Internacionalización de la Universidad guiada desde la lógica del mercado.

A lo largo de la historia se ha hecho referencia a la importancia de la integración de los países latinoamericanos, con el objetivo de buscar solución a problemáticas comunes, así se han implementado distintos acuerdos 0 programas de integración, sin embargo, varios quedaron en la fase declarativa, sin originar actividades concretas. En la última década del siglo $\mathrm{XX}$, surge una nueva iniciativa de integración en el Cono Sur de América Latina, el Mercosur con una impronta fuertemente comercial. No obstante, en los últimos años, se incluyó al debate la necesidad de repensar y profundizar los acuerdos con el fin de construir alianzas estratégicas sobre la base de la solidaridad y complementariedad. Esto permitiría correr el eje de la integración, pasando de una integración guiada por el mercado a una integración con mayor contenido social, pensada por y para los puecado a una integración con mayor contenido social, pensada por y para los pueblos "en diferentes intervenciones los Jefes 
de Estado y de Gobierno han afirmado que el MERCOSUR debe convertirse en un instrumento político y social que consolide a la región como un bloque global en la búsqueda efectiva del multilateralismo y la integración" (Luis Marcano; 2009). Pero ¿Cuál es el rol de las Universidades en el proceso de integración? De acuerdo con Miguel Rojas Mix (2005) se considera que, si estamos convencidos de que nuestro futuro planetario está en la integración, la Universidad debe comprometerse con ese destino en una política de cooperación académica. Incluir en la currícula del futuro la creación de redes temáticas, multidisciplinarias y asociativas de Universidades, destinadas a responder y anticipar los desafíos sociales, a desarrollar la pertinencia de la investigación científica, formando a las nuevas generaciones en concepciones mucho más amplias, que abarquen e integren el conocimiento de la historia, la literatura, la cultura, las ciencias y las artes en estructuras comprensivas de todo el continente latinoamericano. Consecuentemente se enfatiza en la importancia que tiene la investigación de la Universidad Latinoamericana, en relación al desarrollo de los países que la sustentan. Entendiendo al desarrollo de los países por la capacidad en resolver las necesidades de su población, a partir del dominio de las tecnologías, mediante procesos de creación, transferencia y apropiación, teniendo como objetivo la producción de insumos necesarios para la vida digna en condiciones ambientalmente sustentables, esta forma de definir el desarrollo se opone a la atención de patrones de consumo impuestos por el mercado o por otras culturas (Luis Marcano; 2009) De acuerdo a Siufi la integración de las Universidades Latinoamericanas ha avanzado más en términos declarativos que en acciones concretas, la autora sostiene que los proyectos, programas y actividades no se traducen en políticas regulares, ni en prácticas de implementación. Además, muestran 
dificultades en la evaluación de los programas de cooperación, debido a la falta de informes, y de estudios de impacto (Siufi; 2012: 136). Por otro lado, los aportes de Krotsch (1997) y Didriksson (2002), hacen referencia a la importancia de los consorcios de Universidades para la integración universitaria, Krotsh (1997) enfatiza en las problemáticas que trae aparejada la integración educativa, como son: la compatibilidad de títulos, programas y planes de estudio, habilitaciones profesionales y movilidad académica. Si bien muchas de las problemáticas aún no se lograron resolver, se está trabajando en la generación de instrumentos. En el ámbito del Mercosur los instrumentos para orientar la internacionalización hacia la Región se proyectan a partir del "Sector Educativo del MERCOSUR" (SEM) que tiene como objetivo la conformación de un espacio educativo común concertando políticas articuladoras entre la educación y el proceso de integración del MERCOSUR. Así luego de dos décadas de trabajo este espacio ha logrado desarrollar experiencias compartidas de trabajo conjunto y una visión de bloque con enfoque propio en base a objetivos estratégicos (Larrea y Astur; 2011)

"Teniendo en cuenta las deudas pendientes en materia de inclusión y educación de calidad, el siglo XXI nos insta a trabajar arduamente con el objetivo de hacer frente a estos desafíos. En este contexto de fortalecimiento de las políticas de integración regional, la educación juega un papel estratégico, siendo el Sector Educativo del MERCOSUR - SEM, con sus veinte años de existencia y trabajo ininterrumpidos, instrumento esencial para la construcción de un espacio educativo integrado a través de la coordinación de las políticas educativas" (Plan de Acción del SEM; 2011).

\section{Bibliografía}


- Altbach, P. (2004). Globalization and the university: Myths and realities in an unequal World. Tertiary Education and Manangement, (10), 3-25.

- Ben-David, J. (1977). Centers of Learning Britain, France, Germany and the United States. Berkeley, California: The Carnegie Commission on Higher Education.

- Beneitone, P. (2008). Internacionalización del Currículo en América Latina y el Caribe. Foro sobre Internacionalización de la Educación Superior. Llevado a cabo en Universidad del Cauca. Disponible en: http://www.ascun.org.co/eventos/forointernacionalización/pablobeneitone .pdf Bernal, M. (2007). La internacionalización de la Educación Superior. El debate global de los años noventa hasta el presente, y sus futuras implicancias. Córdoba: Editorial de la Universidad Católica de Córdoba (EDUCC).

- Bernal, M. (2007). La internacionalización de la Educación Superior. El debate global de los años noventa hasta el presente, y sus futuras implicancias. Córdoba: Editorial de la Universidad Católica de Córdoba (EDUCC).

- Blanco Moreno, José Luis. (2015) La Proyección Social a través de la gestión de proyectos de impacto. Universidad Santo Tomás

- Brunner, J. (2005). Internacionalización transnacional de la educación superior. Disponible en: http://mt.educarchile.cl/mt/jibrunner/archi ves/JJB internacES.pdf

- Brunner, J. J. (1999). "Educación superior en una sociedad global de la información".

Disponible

en:

http://archivos.brunner.cl/jibrunner/archives/20058.pdf (accedido en septiembre de 2010) 
- Didou Aupetit, S. (2007). La internacionalización de la educación superior en América Latina: Oportunidades y desafíos. In Conferencia dictada en el Pabellón Argentina de la Ciudad Universitaria, Córdoba

- Didriksson, A. (2002). Las Macrouniversidades de América Latina y el Caribe. Venezuela: IESALC-UNESCO.

- Gacel Ávila, J. (2000a). La Internacionalización de las Universidades Mexicanas. Políticas y Estrategias Institucionales. México: ANUIES.

- Gacel-Ávila, J. (2005) La internacionalización de la Educación Superior en América Latina: El caso de México. Cuaderno de Investigación en la Educación 20, Universidad de Guadalajara. México.

- García Guadilla C. (2005). Complejidades de la globalización e internacionalización de la educación superior. Interrogantes para América Latina. Cuadernos del CENDES, 58(22), 1-22.

- Gascón Muro, P. y Cepeda Dovala, J. (2004). De la mercantilización a la transnacionalización de la educación superior. Reencuentro. Análisis de problemas universitarios, (40), 1-15.

- Gascón Muro, P. y Cepeda Dovala, J. (2009). Gascón Muro, P. y Cepeda Dovala, J. (2004). De la mercantilización a la transnacionalización de la educación superior. Reencuentro. Análisis de problemas universitarios, (40), 1-15. 15.

- Knight, J. (1994). Internationalization: Elements and Checkpoints. Monografía Investigativa $N^{\circ} 7$. Oficina de Canadá para la Cooperación Internacional, Otawa.

- Knight, J. (2006) An Internationalization Model: Responding to New Realities and Challenges, in Hans de Wit, et al., Higher Education in Latin America, World Bank, Washington D. C. Págs. 1-39 
- Krotsch, P. (1997). La Universidad en el proceso de integración regional. Perfiles Educativos, XIX(77). Http://www.redalyc.org/html/132/13207711/

- Maria L. Parrales-Poveda; Diego R. Sornoza-Parrales; Rocio J. CanoAndrade; Mercy K. Moreira-Cañart (2018). Una mirada acerca de la comunicación en la Educación Superior. Pol. Con. (Edición núm. 20) Vol. 3, No 6, pp. 277-289, ISSN: 2550 - 682X

- Oregioni, M. (2013a). Aspectos Político Institucionales de la Internacionalización de la Universidad Nacional de La Plata. Revista Argentina de Educación Superior, 5(6), 97-118.

- Oregioni, M. (2013b). La Universidad como actor de la Cooperación SurSur. El caso de la Universidad Nacional de La Plata en la Asociación de Universidades del Grupo Montevideo Disponible en: http://revistas.unc.edu.ar/index.php/integracionyconocimiento/article/view $15893 / 6911$

- Oregioni, M. (2014). Dinámica de la Internacionalización de la Investigación en la Universidad Nacional de La Plata (2005-2012). Tesis Doctoral en Ciencias Sociales y Humanas. Universidad Nacional de Quilmes, Buenos Aires

- "Oregioni, M. et. al. (2014). Proyección integral de la universidad hacia la Región Latinoamericana. En D. Herrero y B. Dalmolin, Libro de las Jornadas Extensión Mercosur. Buenos Aires: Universidad Nacional del Centro de la Provincia de Buenos Aires."

- Perrota, D. (2011). La integración regional de la educación superior en el MERCOSUR en el marco de la orientación general del bloque y la tensión entre un modelo solidario y otro competitivo: el caso de los programas regionales de acreditación de carreras de grado (del MEXA al 
ARCU-SUR, 1998-2008) (Tesis de Maestría). FLACSO. Buenos Aires. Argentina.

- Perrotta, D, (2016) La internacionalización de la universidad. Debates globales, acciones regionales, IEC-UNGS, Los Polvorines

- Ruiz, C. (2014). Internacionalización de la educación Superior paradiplomacia universitaria y la gestión de la comunicación en este proceso. Tesis de grado no publicada de Comunicación. Universidad de Piura. Facultad de Comunicación. Piura, Perú.

- Tauber F. (2018). Pensar la Universidad Publicación Institucional de la Universidad Nacional de La Plata

- Theiler, J. (2005). Internacionalización de la educación Superior en Argentina. En J. Knight, J. et. al., Educación Superior en América Latina. La dimensión internacional (pp. 71-112). Argentina: Banco Mundial.

- Vessuri, H. (2007). O inventamos o erramos. La ciencia como ideafuerza en América Latina. Ciencias, (102), 58-59.

- Vessuri, H. (2013). El nuevo "mantra" de la diplomacia científica internacional: ¿Co-diseño de conocimiento? ¿Investigación integrativa? Universitas Humanística, (76), 25- 55.

- Yarzábal, L. (2005). Internacionalización de la Educación Superior: de la cooperación académica al comercio de servicios. En J. Pugliese (Ed.), Educación superior ¿Bien público o bien de mercado? Los debates sobre la Internacionalización desde los noventa a la actualidad (s/d). Buenos Aires: MECyT-SPU. 